\title{
Papillon Lefevre Syndrome: Diagnosis and Management in Two Affected Siblings - A Case Report
}

$\begin{array}{ll}\text { Abul Khair Zalan } & \text { BDS } \\ \text { Khadeejah Khalil Zubairy }^{2} & \text { BDS } \\ \text { Anser Maxood }^{3} & \text { BDS, FRACDS } \\ \text { Manahil Niazi }^{4} & \text { BDS } \\ \text { Hira Zaman }^{5} & \text { BDS, MDS } \\ \text { Anika Gul }^{6} & \text { BDS } \\ \text { Miraat Anser }^{7} & \text { BDS }\end{array}$

Papillon-Lefèvre syndrome (PLS) an autosomal recessive disorder characterized by diffuse transgradient palmar-plantar hyperkeratosis, with premature loss of deciduous and permanent teeth, along with the calcification of the dura mater. This results in teeth radiographically appearing as "floating" in the soft tissue. Genetic studies of patients with PLS have mapped the major gene locus to chromosome 11q24-q21 and revealed mutation and loss of function of the cathepsin gene. It affects 1- 4 people per 1 million population with no gender or racial predilection. Dermatological manifestations, usually occurs before four years of age, include hyperkeratosis of palms and soles, nail dystrophy, hyperhidrosis and keratinization on elbows and knees with the lesions appearing as white, yellow-like or red plaques or patches that then develop cracks, crusts, or deep fissures. KEYWORD: Papillon-lefevre syndrome, hyperkeratosis

HOW TO CITE: Zalan AK, Zubairy KK, Maxood A, Niazi M, Zaman H, Gul A, Anser M. Papillon lefevre syndrome: Diagnosis and management in two affected siblings - A case report. J Pak Dent Assoc 2020;29(4):264-268.

DOI: https://doi.org/10.25301/JPDA.294.264

Received: 23 May 2020, Accepted: 24 August 2020

\section{INTRODUCTION}

$\mathrm{P}$ apillon-Lefèvre syndrome (PLS), was first described by two physicians, Papillon and Lefèvre, in France, 1924. ${ }^{1}$ It is an autosomal recessive disorder characterized by diffuse transgradient palmar-plantar hyperkeratosis, with premature loss of deciduous and permanent teeth, along with the calcification of the dura mater. ${ }^{2}$ Genetic studies of patients with PLS have mapped the major gene locus to chromosome 11q24-q21 and revealed mutation and loss of function of the cathepsin gene. ${ }^{3}$ It affects

1. MDS (Resident), Department of Pediatric Dentistry, Children Hospital, Pakistan Institute Medical Sciences, Islamabad.

2. House Surgeon, Department of Pediatric Dentistry, Children Hospital, Pakistan Institute Medical Sciences, Islamabad.

3. Dean of Dentistry, Head of Department, Department of Operative and Pediatric Dentistry, Pakistan Institute Medical Sciences, Islamabad.

4. House Surgeon, Department of Prosthodonti, Armed Institute of Dentistry, Rawalpindi.

5. Registrar, Department of Operative Dentistry, University College of Dentistry, University of Lahore.

6. FCPS (Resident) Department of Orthodontics, Sardar Begum Dental College, Peshawar.

7. MDS (Resident), Department of Pediatric Dentistry, Children Hospital, Pakistan Institute Medical Sciences, Islamabad.

Corresponding author: “Dr. Abul Khair Zalan” < zalanjan@yahoo.com >
1- 4 people per 1 million population with no gender or racial predilection. With the eruption of deciduous teeth, diffuse hemorrhagic and hyperplastic gingivitis along with the loss of periodontal attachment develops. This results in teeth radiographically appearing as "floating" in the soft tissue. By 4 to 5 years of age, all primary teeth are lost or extracted with the gingiva returning to normal state of health until the eruption of permanent teeth. The permanent teeth also start exfoliation just after eruption and are lost by the age of 14-15. There is dramatic alveolar bone destruction, often leaving atrophied jaws. Dermatological manifestations, usually occurs before four years of age, include hyperkeratosis of palms and soles, nail dystrophy, hyperhidrosis and keratinization on elbows and knees with the lesions appearing as white, yellow-like or red plaques or patches that then develop cracks, crusts, or deep fissures. The exact etiopathogenesis of this syndrome is not clearly understood and genetic, bacterial, or possible immunologic etiologies have been proposed. Many microbiologic studies and clinical examinations after different treatment protocols have established a close association between the occurrence of 
Zalan AK/ Zubairy KK/ Maxood A/

Niazi M/ Zaman H/ Gul A/ Anser M

Actinobacillus actinomycetemcomitans and destruction of periodontium in patients with PLS. ${ }^{4}$

\section{CASE REPORT}

A 14 years old male and his brother, a 13 years old male, otherwise healthy individuals, were seen at the outpatient department of Pediatric dentistry, children hospital, Pakistan Institute of Medical Sciences (PIMS), Islamabad. The presenting complaint of both the patients was falling of teeth immediately after their eruption. Further history revealed that the parents of the patients had a primary consanguineous marriage and a positive history of early shedding of teeth in the family. For elaboration, the cases are numbered as 1 and 2.

\section{CASE 1}

On extraoral examination, the 14 years old male had normal facial features and decreased facial height with hyperkeratosis on palms and feet. Intraoral examination revealed compromised oral hygiene with accumulation of plaque and food debris around the tooth surfaces in the mandibular arch while the maxillary arch was edentulous with markedly resorbed alveolar bone. A total number of

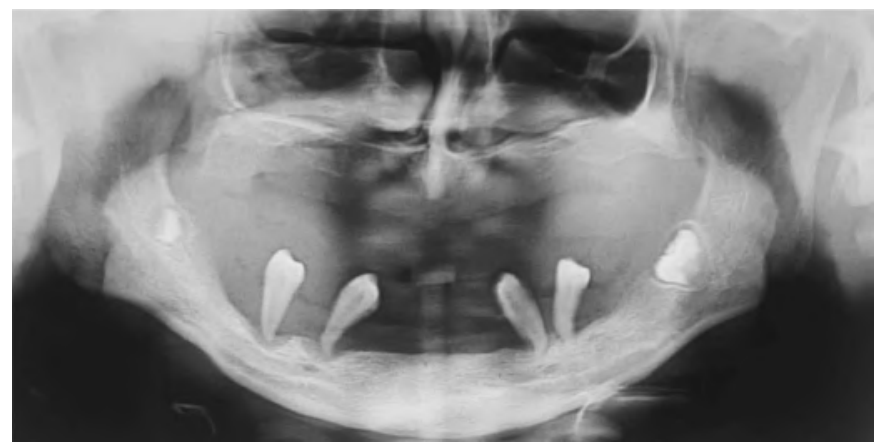

Fig.1: Orthopantamogram showing generalized alveolar bone loss to apical third of roots, Mandibular canines and first premolars in both quadrants are almost out of the socket (floating in air appearance) with little or no bony support. Mandibular third molars found erupting in both quadrants. Resorbed and completely edentulous maxillary ridge can also be seen.

four teeth were present in the mandible which were canines and first premolars on both sides of the arch. Aggressive Periodontitis was present and all of the teeth had Grade III mobility (according to miller tooth mobility index 1985) which seemed to be "floating in the soft tissue" because of severe bone loss and exposed cementum. OPG radiograph was advised, which showed that the four teeth present in the mandibular arch had only 1-2 mm of bone support while third molars had not erupted yet. A complete blood picture was advised which was normal. On the basis of detailed

\section{Papillon Lefevre syndrome: Diagnosis and management in two affected siblings - A case report}

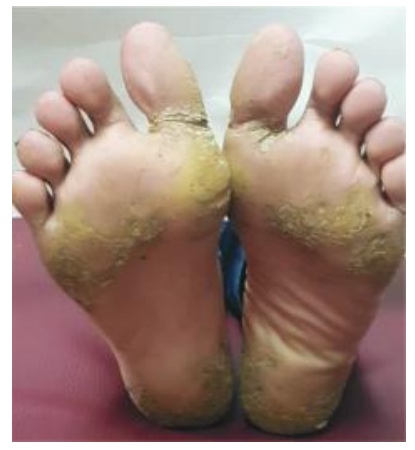

Fig.2: Showing yellow colored crusted and cracked hyperkeratotic regions on sole.

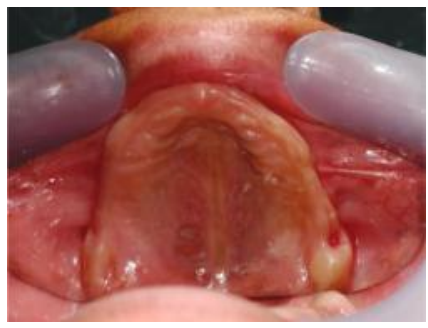

Fig.4: Intraoral view showing edentulous maxilla

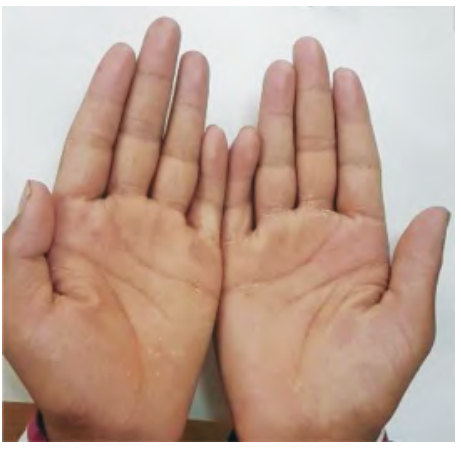

Fig.3: Hyperkeratosis of palms

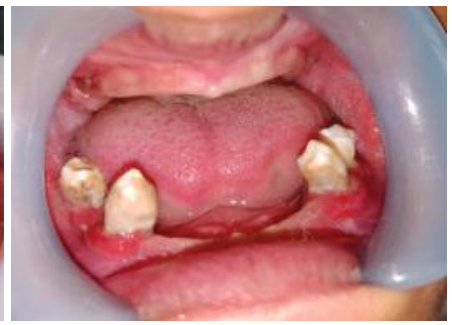

Fig.5: Intraoral view showing canines and first premolars with exposed cementum and with severe periodontitis.
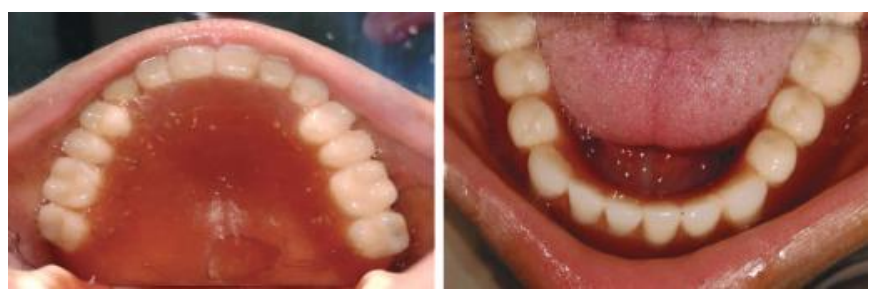

Fig.6: Showing acrylic complete denture in both arches.
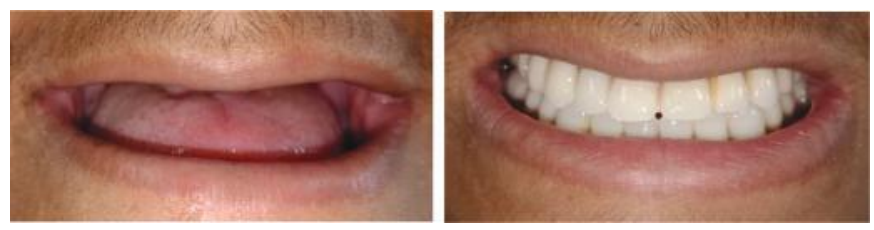

Fig.7: Extraoral view showing before and after the insertion of complete denture in both maxilla and mandible

family history, clinical and radiographic evaluation, the diagnosis of Papillon lefevere syndrome (PLS) was confirmed. Consanguineous marriages is considered as a high risk factor for this disease. ${ }^{11}$

Treatment was started with instructions for the improvement of oral hygiene and $0.12 \%$ chlorhexidine was prescribed for two weeks to decrease the bacterial load. Because of relatively late presentation to the dental department as compared to his younger brother, all of the teeth present in the mandibular arch were already loosened due to 
Zalan AK/ Zubairy KK/ Maxood A/

Niazi M/ Zaman H/ Gul A/ Anser M

aggressive periodontitis which was accompanied with poor bone support and grade III mobility, hence considered nonsalvageable and were extracted. Patient was recalled after six weeks to re-evaluate the oral health and healing gums and soft tissues. The oral health and healing of gums was considered satisfactory. Provision of complete denture was planned. Primary impression was taken in alginate, a cast was formed. Special tray was made from the cast with acrylic to take secondary impression with impression compound. Base plate was formed from the cast made up of secondary impression. Patient was subsequently recalled to take occlusal vertical dimension, trial and final insertion. Patient was then referred to dermatologist for the treatment of associated skin involvement. Follow up of one year showed good compliance and improved general health. Third molars were still not erupted. Implant retained prosthesis will be given after growth completion along with synthetic bone graft placement because of severely resorbed maxillary and mandibular alveolar bone with deficient alveolar bone heights.

\section{CASE 2}

Extra-oral examination of 13 years old male patient showed normal facial features with hyperkeratosis of palms and soles. Intraoral examination revealed compromised oral hygiene with accumulation of plaque and food debris around the teeth present with marginal gingivitis. Loss of multiple teeth were seen in maxillary and mandibular arch. Exfoliated maxillary teeth were left central incisor, right second premolar

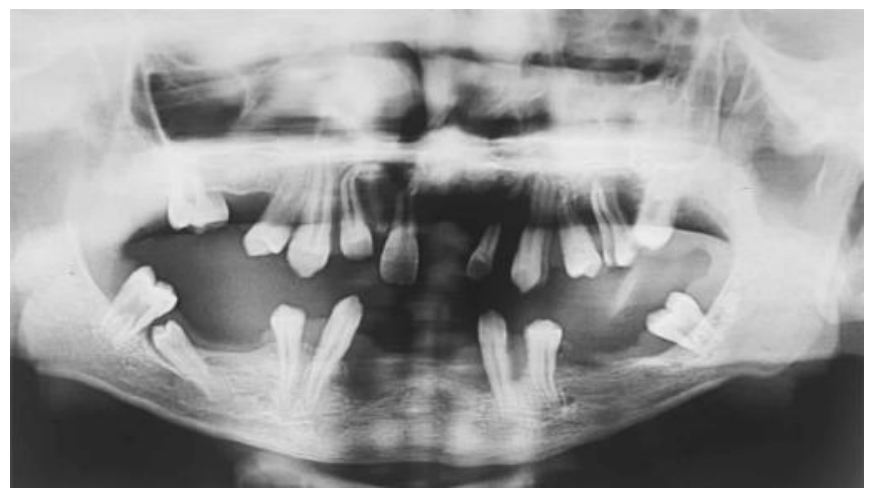

Fig.8: Radiograph showing generalized destruction of alveolar bone and multiple missing teeth in both upper and lower jaw.

and second and third molars on both sides, while exfoliated mandibular teeth were central incisors, lateral incisors, left second premolar and bilateral first and third molars. Multiple tilted/ drifted teeth were also present, although with no pathological mobility. OPG (orthopantomogram) radiograph was advised, which showed impacted mandibular right second premolar. Complete blood picture was advised which showed no findings. On the basis of detailed family history,

Papillon Lefevre syndrome: Diagnosis and management in two affected siblings - A case report

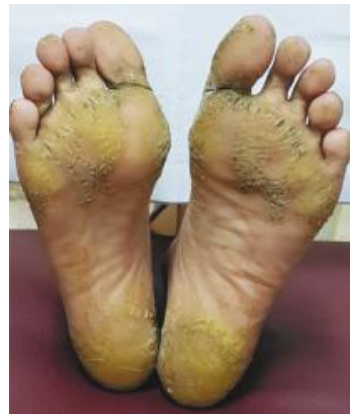

Fig.9: Dermatologic manifestation in form of plantar hyperkeratosis

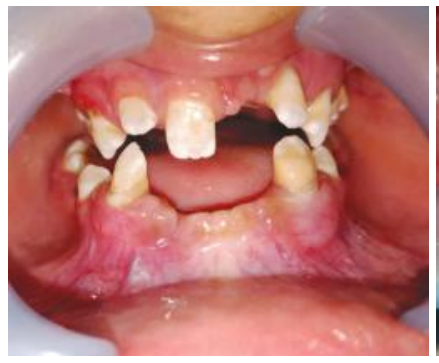

Fig.11: Intraoral view showing missing left central incisor in maxilla and multiple missing teeth in mandible.

extra-oral and intra-oral clinical and radiographic evaluation, a diagnosis of PLS was confirmed.

Treatment was started with the instructions for the improvement of oral hygiene and $0.12 \%$ chlorhexidine gluconate mouth rinse prescription for two weeks. Patient was then recalled for scaling and root planning. A provision of removable partial dentures was planned for the exfoliated maxillary and mandibular teeth. Acrylic dentures were made for both arches. After insertion of dentures, maintenance of meticulous oral hygiene instructions were was not given to prevent the accumulation of pathological microbes which aggravate periodontal breakdown and teeth loss. Patient was referred to the dermatology department for the treatment of cutaneous lesions. More frequent recall schedule of 3-6 months was planned for scaling/root planning along with the evaluation of oral hygiene and periodontal health and repeatedly patient and parental counselling for maintenance of vigorous oral hygiene care to delay exfoliation of primary and permanent teeth till growth completion. Delaying shedding of teeth until growth completion helps in the attainment of optimal 
Zalan AK/ Zubairy KK/ Maxood A/

Niazi M/ Zaman H/ Gul A/ Anser M

alveolar bone height, which negates bone graft surgeries during placement of implant supported prosthesis. A good compliance with improved general health was noticed after one year of follow-up. Implant retained prosthesis will be given after growth completion.

\section{DISCUSSION}

The exact etiology and subsequent development of the Papillon lefevere syndrome is still not clear. Multiple factors including immunologic, microbiologic, and genetic appearance to be involved in the causation of PLS. Dysfunction of neutrophils (immunological) appearing as decreased phagocytic and chemotactic activities leading to deficient function of the monocytes, depression of helper/suppressor $\mathrm{T}$ cells ratio and decreased lymphocyte response to pathogens are considered to attribute in the development of this syndrome. ${ }^{5}$ Thus, increased susceptibility to infections is a common finding in this syndrome. The contributing organisms (microbiological) that have been established in PLS are Actinobacillus actinomycetemcomitans, Fusobacterium nucleatum, Porphyromonas gingivalis and Treponema denticola. Various studies have proposed that the mutation of the cathepsin-C gene (genetic) is also associated with the dental and dermatological abnormalities observed in this syndrome. This genetic mutation is inherited as an autosomal recessive pattern. With both of the parents as carriers of the defective gene, the risk increases up to $25 \% .{ }^{8}$ Consanguineous marriage is also contributory to PLS and considered as the high risk factor, which was observed in our case.11 Hence, familial counselling regarding genetics is very important in our country because of $20-50 \%$ of consanguineous marriages of total population. ${ }^{12,13}$

Dermatological and Periodontal changes basically characterizes Papillon Lefevre Syndrome. Cutaneous lesions include sharply demarcated palmoplantar hyperkeratosis while the periodontal changes includes severe aggressive periodontitis resulting in early loss of primary and permanent dentition, painful gums and multiple periodontal abscesses. Ullbro et al. did not observe any significant correlation between the severity of skin involvement and the severity of the periodontal infection. ${ }^{9}$ In the current case all these dental findings and skin lesions were observed and attention has been given to the timely treatment and management of the syndrome for the better outcome in the later life.

Other clinical features in PLS includes calcification of the dura mater, tentorium cerebelli, choroids plexus and falx cerebri. Intra-abdominal abscesses (pyogenic liver abscess) have also been reported. ${ }^{7}$ We could not observe any of these in the current case.
Papillon Lefevre syndrome: Diagnosis and management in two affected siblings - A case report

Greither's syndrome and Howel-Evans syndrome, acrodynia, cyclic neutropenia, hypophosphatasia, are the conditions which share similar signs and symptoms with PLS except periodontitis, so they were ruled out. ${ }^{1}$

Haim-Munk syndrome is an allelic variation of PLS but it shows additional clinical features including arachnodactyly and acro-osteolysis which are not present in PLS. ${ }^{6}$

The treatment plan necessitates multidisciplinary approach involving dermatologists and dental surgeon team (Pediatric dentists, Periodontists, Prosthodontists). Pediatric dentists should first recognize and timely manage patients with this syndrome for better outcomes in the later life after growth completion. Early diagnosis and prompt dental treatment guarantee better prognosis.

For the treatment of dental manifestations, No specific treatment has been proposed but the main aim is to vigorously improve oral hygiene, eliminate the reservoir of micro-organisms and limit the periodontal destruction and allow teeth to be present and delay permanent teeth exfoliation until growth completion and attainment of optimal alveolar bone height. For this purpose, several modalities including conventional periodontal treatment (scaling and root planning); along with extraction of teeth with severe periodontitis and Grade-III mobility, followed by prosthodontic replacement is considered one of the mainstays of treatment. This was the treatment option adopted in our case. Oral hygiene instructions, a prescription of $0.12 \%$ chlorhexidine gluconate mouth rinses and more frequent recalls for professional teeth cleansing and root planning along with one-week systemic antibiotics to control aggressive periodontitis and to prevent bacteremia and pyogenic liver abscesses should also be considered. Early extraction of non-salvageable teeth has also been recommended to prevent further periodontal destruction and bone loss thus allowing protection of a solid base for implantation of artificial dentures. ${ }^{10}$

Oral retinoids like isotretinoin, acitretin, and etretinate have also been suggested for the treatment of both the dental and the cutaneous defects observed in the PLS ${ }^{7}$, but they show many adverse effects and hence not used in our study. Treatment with retinoids before the eruption of permanent teeth results in a normal dentition, and is more efficacious if continued during the development of permanent teeth. PLS may also adversely affect the self-esteem and general health of children. So, a multidisciplinary approach addressing the social and psychological needs of these children is also necessary. Dental and dermatological evaluation and its early diagnosis and treatment by pediatric dentist along with parental counseling regarding the genetics are an important part of the management strategy. Maintenance of good periodontal health is imperative in the management of PLS 
Zalan AK/ Zubairy KK/ Maxood A/

Niazi M/ Zaman H/ Gul A/ Anser M

patients. So, a regular follow up of 3-6 months for ultrasonic scaling and evaluation and maintenance of vigorous oral hygiene is necessary to prevent microbial accumulation, periodontal breakdown and early teeth loss. Teeth loss before growth completion results in immature alveolar bone with marked alveolar ridge resorption, needs temporary restorations till adulthood and requires surgical bone grafting for final prosthesis.

\section{CONCLUSION}

Pediatric dentists should timely diagnose and manage patients with papillon lefevre syndrome.

\section{CONFLICT OF INTEREST}

None declared

\section{REFERENCES}

1. Sreeramulu B, Shyam ND, Ajay P, Suman P. Papillon-Lefèvre syndrome: clinical presentation and management options. Clin Cosmet Investig Dent. 2015;7:75-81. https://doi.org/10.2147/CCIDE.S76080

2. Gorlin RJ, Sedano H, Anderson VE. The syndrome of palmarplantar hyperkeratosis and premature periodontal destruction of the teeth. A clinical and genetic analysis of the Papillon-Lefèvre syndrome. J Pediatr. 1964;65:895-08 https://doi.org/10.1016/S0022-3476(64)80014-7

3. Hart TC, Hart PS, Bowden DW, et al, Mutations of the cathepsin $\mathrm{C}$ gene are responsible for Papillon-Lefèvre syndrome, J Medi Geneti 1999;36:881-87

4. Alsarheed M, Al-Sehaibany F. Combined orthodontic and periodontic treatment in a child with PapillonLefèvre syndrome. Saudi Med J. 2015;36:987-92

https://doi.org/10.15537/smj.2015.8.11437
Papillon Lefevre syndrome: Diagnosis and management in two affected siblings - A case report

5. Singla A, Sheikh S, Jindal SK, Brar R. Papillon Lefevre syndrome: Bridge between dermatologist and dentist. J Clin Exp Dent 2010;2:e436

https://doi.org/10.4317/jced.2.e43

6. Tasli L, Kacar N, Erdogan BS, Ergin S. Successful treatment of Papillon Lefevre syndrome with a combination of acitretin and topicalPUVA; a four year follow up. J Turk Acad Dermatol. 2009;3:1-4.

7. Pavankumar K. Papillon-Lefèvre syndrome: A case report. Saudi Dent J 2010;22:95-98

https://doi.org/10.1016/j.sdentj.2010.02.009

8. Hart TC, Shapira L. Papillon-Lefèvre syndrome. Periodontol 2000. 1994;6:88-100.

https://doi.org/10.1111/j.1600-0757.1994.tb00029.x

9. Ullbro C, Crossner CG, Nederfors T, Alfadley A, Th estrup-Pedersen K Dermatologic and oral findings in a cohort of 47 patients with Papillon-Lefèvre syndrome. J Am Acad Dermatol 2003;48:345-51. https://doi.org/10.1067/mjd.2003.197

10. Janjua S.A., Khachemoune A. Papillon-Lefevre syndrome: case report and review of the literature. Dermatol. Online J. 2004;10:13

11. Shah AF, Tangade P, Agarwal S. Papillon-Lefevre syndrome: Reporting consanguinity as a risk factor. The Saudi Dent J. 2014;26: 126-31.

https://doi.org/10.1016/j.sdentj.2014.02.004

12. Modell B, Darr A. Science and society: genetic counselling and customary consanguineous marriage. Nat Rev Genet. 2002;3:225-9. https://doi.org/10.1038/nrg754

13. Vardi-Saliternik R, Friedlander Y, Cohen T.Consanguinity in a population sample of Israeli Muslim Arabs, Christian Arabs and Druze. Ann Hum Biol. 2002;29:422-31.

https://doi.org/10.1080/03014460110100928 